\title{
Circuit
}

Musiques contemporaines

\section{Nouveautés en bref}

\section{Réjean Beaucage}

Volume 17, numéro 3, 2007

Musique in situ

URI : https://id.erudit.org/iderudit/017598ar

DOI : https://doi.org/10.7202/017598ar

Aller au sommaire du numéro

Éditeur(s)

Les Presses de l'Université de Montréal

ISSN

1183-1693 (imprimé)

1488-9692 (numérique)

Découvrir la revue

Citer ce compte rendu

Beaucage, R. (2007). Compte rendu de [Nouveautés en bref]. Circuit, 17(3),

125-128. https://doi.org/10.7202/017598ar

Ce document est protégé par la loi sur le droit d'auteur. L'utilisation des services d'Érudit (y compris la reproduction) est assujettie à sa politique d'utilisation que vous pouvez consulter en ligne.

https://apropos.erudit.org/fr/usagers/politique-dutilisation/
Cet article est diffusé et préservé par Érudit.

Érudit est un consortium interuniversitaire sans but lucratif composé de l’Université de Montréal, l'Université Laval et l'Université du Québec à Montréal. Il a pour mission la promotion et la valorisation de la recherche. https://www.erudit.org/fr/ 


\section{Nouveautés en bref}

Réjean Beaucage

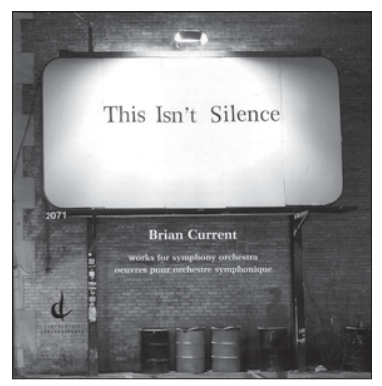

Brian Current: This Isn't Silence

Works for symphony orchestra/

œuvres pour orchestre symphonique-

Centrediscs/Centredisques, 2007,

CMCCD 12607

Un disque canadien regroupant cinq œuvres orchestrales d'un jeune compositeur (1972) est une rareté (on est tenté d'écrire «une anomalie») à saluer. Originaire d'Ottawa, Brian Current a étudié la musique à l'Université McGill (Montréal), auprès de John Rea et Bengt Hambreaus, avant de compléter un doctorat en composition à Berkeley (Californie). Le présent disque regroupe toutes ses œuvres pour orchestre (le Concertino for Flutes ङ String Orchestra [2002, rév. 2004] étant pour «grand ensemble», ici, les cordes de l'ensemble New Music Concerts, sous la direction du compositeur).

La musique de Current est vive, énergique et, par moments, même agressive. Dès l'ouverture, avec la pièce-titre (1998, rév. 2001), l'auditeur a l'impression d'arriver en pleine séance de torture, les cordes et les percussions martelant à l'unisson tandis que quelqu'un pousse des cris à contretemps. This Isn't Silence, en effet! Les choses n'en restent pas là, mais il y a dans tout ce qui suit beaucoup de pizzicato, de grincements divers, de petits cris et une joyeuse luxuriance au sein de l'orchestre (Winnipeg Symphony Orchestra/ Andrey Boreyko, pour cette pièce).

Vient ensuite For the Time Being (CBC Radio Orchestra/Bramwell Tovey), une œuvre qui n'est sans doute pas étrangère à la possibilité même de produire ce disque, puisque c'est grâce à elle que le compositeur a obtenu en 
2000 le prix du jury et le prix du public au Forum international pour les jeunes compositeurs du Nouvel Ensemble Moderne (enregistrement disponible sur le disque «Forum 2000/2002»; NEM/Lorraine Vaillancourt, paru chez ATMA); c'est aussi cette pièce qui remportait en 2001 le grand prix du Concours national des jeunes compositeurs de la Société Radio-Canada.

La présentation chronologique des œuvres permet d'apprécier l'évolution du compositeur à travers l'assurance que gagne son écriture. Il est surtout réjouissant de constater qu'entre la première œuvre et les plus récentes (Kazabazua [2003, rév. 2006] et Symphonies in Slanted Time [2005], toutes deux interprétées par l'Esprit Orchestra et Alex Pauk), le tranchant ne s'émousse pas et le grain reste à certains endroits assez rude, ou dur; le compositeur sait jouer de la masse orchestrale et il ne s'en prive pas. Voici donc un disque qui ne nuira certes pas à la carrière de Current et qui risque de faire beaucoup d'envieux parmi les jeunes compositeurs canadiens.

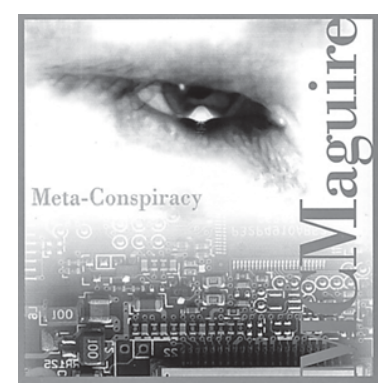

MC Maguire : Meta-Conspiracy Tzadik, 2007, 8034

Si j’ai évoqué une certaine agressivité à propos de la musique de Brian Current, dans le cas de Michael C. Maguire, on peut carrément parler de violence, du type «Massacre à la tronçonneuse »... Dans une «déclaration de l'artiste»

inscrite sur son site Internet, le compositeur explique: «As a composer I have created a very personal post-modern hybrid which is a quirky synthesis of the historical and modern western tradition, as well as combining styles of popular music and the various primitive and high cultural traditions of world music»; ce qui n'est pas dit ici, c'est qu'il fait tout ça simultanément! Enfin, presque. La musique de Maguire évoque immédiatement celle de l'électroacousticien Paul Dolden, qui construit ses œuvres en accumulant des centaines de pistes, éventuellement d'un même instrument, pour donner au son d'ensemble une texture et une profondeur incomparables (enfin, jusqu'à maintenant). Les œuvres de Maguire comptent jusqu'à 400 pistes, et, de plus, elles sont mixtes: A Short History of Lounge (2001-2006) est écrite pour piano (David Swan) et ordinateur (for piano and cpu), tandis que Got that Crazy, Latin/Metal Feelin' (2003-2006) est écrite pour guitare électrique (John Gzowski) et ordinateur (on aimerait entendre la version originale pour petit ensemble, guitare électrique et ordinateur, une commande de Vancouver New Music). 
Malgré son titre, A Short History of Lounge n'évoque ce genre musical qu'à travers une ligne de basse vaguement jazzée qui revient tout au long de l'œuvre par bouffées (Maguire parle d'une rumba boulézienne); l'ensemble, outre certains passages où le piano est à découvert, distille une énergie proche de celle du rock, et rappelle à l'occasion le type de déflagration sonore propre à quelques œuvres de Xenakis. Cependant, si la première écoute risque fort de déclencher un mal de tête, les écoutes subséquentes permettent de traverser le brouillard qui entoure la musique. La piano s'éclaircit, on croise un orchestre symphonique dans un fragment de ce qui semble être du Bruckner, et on distingue mieux le travaille époustouflant d'assemblage de la multitude de pistes où s'entrecroisent diverses qualités de sons électroniques. Bref, la sensastion d'écouter 12 stations de radio simultanément s'amenuise pour permettre à l'auditeur courageux d'appréhender l'imaginaire multipistes du compositeur.

L'autre œuvre au programme est de la même eau, mais avec un peu plus de raffinement, ou, enfin, un chaos moins forcené. L'utilisation de la guitare électrique et des voix la rapproche à de nombreux moments du style de rock progressif «symphonique» qu'on trouve chez nombre de formations européennes (on pense à Yes, particulièrement), davantage que du heavy metal auquel le titre renvoie.

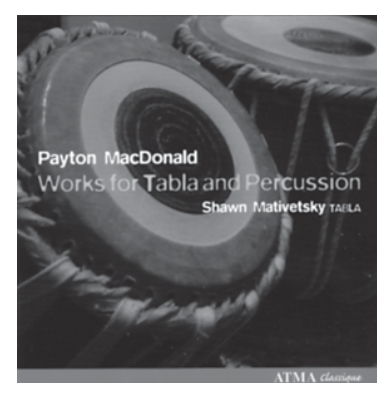

Payton MacDonald :

Works for Tabla and Percussion

Shawn Mativetsky, tabla ;

William Paterson University

Percussion Ensemble

ATMA Classique, 2007, ACD2 2393

Le projet du compositeur étatsunien Payton MacDonald n'est ni le premier ni, sans doute, le dernier des exercices visant à fondre les cultures de l'Est et de l'Ouest en un nouvel alliage multiculturel. De Debussy à Gilles Tremblay, et des Beatles à Led Zeppelin, ceux qui font la musique en Occident ont souvent été fascinés par les rythmes complexes et les harmonies étranges de l'Orient. Les véritables mutations sont rares, et les placages d'une culture sur l'autre n'ont pas souvent plus d'impact sur l'âme de la musique qu'un simple tatouage peut en avoir sur celui qui le porte. On ne peut guère s'interroger très longtemps sur l'authenticité du sentiment qui anime les deux protagonistes principaux de ce programme: Payton MacDonald est percussionniste au sein de l'ensemble Alarm Will Sound, l'une des formations les 
plus intéressantes à avoir vu le jour ces dernières années. Basé à New York, AWS a fait paraître jusqu'à maintenant trois disques consacrés à la musique de Steve Reich et un quatrième qui regroupe des transpositions pour ensemble de la musique électronique d'Aphex Twin. MacDonald est diplômé en percussion et en composition (Université du Michigan et Eastman School of Music) et poursuit l'apprentissage du tabla auprès de Pandit Sharda Sahai, comme Shawn Mativetsky. Ce dernier détient une maîtrise en musique de l'Université McGill, où il enseigne et dirige le McGill Tabla Ensemble.

Le disque offre trois concertos pour tabla et quatuor de percussions $\left(n^{\circ} 1\right.$, 2002; $n^{\circ} 2,2003 ; n^{\circ} 3,2004$ ) et trois courtes pièces pour tabla solo. C'est dans le Concerto $n^{\circ} 3$ que le compositeur réussit le mieux à s'affranchir du poids culturel de son instrument soliste, même si la partie n'est pas gagnée pour l'auditeur, le tabla ayant un son si distinctif, si fortement connoté, que la fusion culturelle est difficile à opérer, d'autant plus que le compositeur respecte "pour l'essentiel la forme d'un solo de tabla classique du style de Bénarès » (extrait du livret d'accompagnement). Reste un répertoire qui met de l'avant le grand talent d'un soliste exceptionnel et une musique qui cherche à établir un pont; l'ouvrage est encore fragile, mais il sera un jour très fréquenté, et on sera alors reconnaissant à ceux qui en ont jeté les bases. 